\title{
Electromagnetic Harbinger Earthquakes
}

\author{
V. K. Balkhanov \\ Institute of Physical Materials Science, Siberian Branch of the Russian Academy of Sciences, Ulan-Ude City, Russia \\ Email: ballar@yandex.ru
}

\begin{abstract}
The radiation of the electric dipole has been calculated, located in the lithosphere and oriented normal to the surface of the Earth. This radiation, in the form of a side wave, reaches the boundary of the atmosphere and lithosphere, and further through the air reaches the measuring complex. This radiation may serve as a harbinger of the earthquake. Offered a method of detecting the electromagnetic Harbinger earthquakes. The measuring complex consists of a vertical electrical antenna and toroidal magnetic antennas located on the Earth's surface. It has been established that the measuring complex is most sensitive to the radiation of the electric dipole, located in the lithosphere array and oriented normally to the Earth's surface. The measuring complex records electromagnetic Harbingers. Detected signal has a number of features that are different from other radiation that allows believe it is a Harbinger of the Earthquake.
\end{abstract}

Keywords: electromagnetic Harbinger Earthquake, dipole, lithosphere, lateral wave.

\section{Introduction}

It is noted in the literature: "Mankind has not learned to predict earthquakes ...", for example, [1]. According to the Seismological community America, application method of forecasting, which was confirmed as a faithful, must ensure good a certain area of the epicenter, the range of time in which this event occurs, and the likelihood that it really happens. The data on which the forecast is based, must be verifiable, and the result of their processing should be repeatable. Earthquake preceded by anomalies of electrical and magnetic fields, emissions of radon gas, helium and other gases [2-7]. Such portents are being observed is not always and everywhere, their combination and the duration is very volatile, and the physical nature is not clear. A promising method of prediction is a solenoid harbinger of earthquakes. In the work [8] the unit had first been proposed to search for it. It is located on the ground the vertical antenna for reception of an electric field, and magnetic frame of the ferrite core in the form of the Torah for the reception of the magnetic field, as shown in Figure 1.

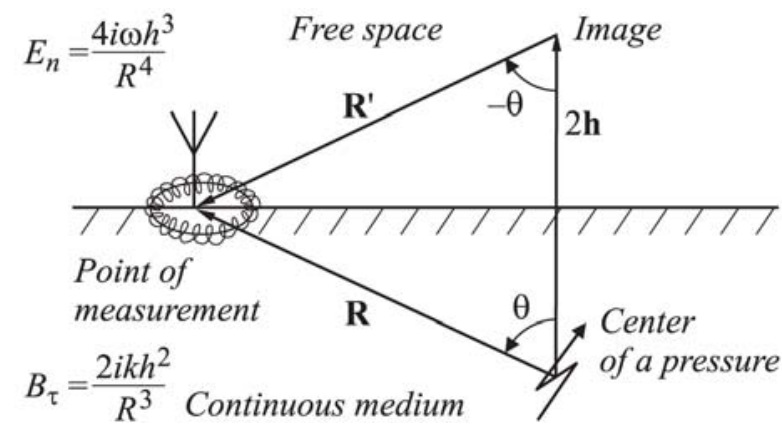

Figure 1. Geometry tasks. In paragraph measuring device is located, electromagnetic field detection. The device consists of an electric and a magnetic antenna vertical frames, wound on a toroidal ferrite core, showing non-zero components of tangential magnetic induction and the normal electric field. Field components have a common factor $\frac{\mu_{0} J l}{4 \pi} \exp (-i \omega t+i k R)$. 


\section{Three Features of the Electromagnetic Precursor}

Received signal must satisfy three conditions. Radiation of electric dipole located in the lithosphere and oriented normal to the surface media, has a number of features that can serve as criteria exactly electromagnetic Harbinger earthquakes. The first criterion follows from the existence of border section "atmosphere-surface". There is a dedicated direction is normal to the border, along which will be sent to delivered from bowels of the Earth at its surface electric field. Magnetic field orthogonally electric field, and due to symmetry polarized in a circle on the Earth's surface. Of the considered the first criterion should be that the alleged electromagnetic Harbinger needs to detect vertical electrical and magnetic antenna in the form of framework, situated also vertically on the surface of the Earth. To amplify the signal from the magnetic field of a system framework, gathered around the circumference. The best electric wire wound on ferrite rod in the form of a toroidal coil, which is located on the surface.

The second criterion electromagnetic Harbinger is the frequency on which you want to measure. To resolve this issue, use the idea of the skin layer. Distribution of electromagnetic field in solid media characterized by skin layer $H$. If $\rho$ - electrical resistivity of homogeneous media, the skin layer [9]

$$
H=\sqrt{\frac{2 \rho}{\mu_{0} \omega}},
$$

where is $\mu_{0}$ - magnetic constant, $\omega$ - circular frequency. The frequency of the electromagnetic field for the surface of the Earth, shall be such that the skin layer of equal depth $h$ occurrence of earthquake. For granitic rocks are usually $\rho \sim 10^{5} \mathrm{Ohm} \cdot \mathrm{m}$ [10]. Having taken for depth $h=10 \mathrm{~km}$, formula (1) find your frequency $f=\omega / 2 \pi$.

$$
f=\frac{\rho}{\pi \mu_{0} h^{2}} \sim 250 \mathrm{~Hz}
$$

Because the depth of the epicenter of the earthquake is unknown in advance, it is necessary to measure broadband spectrum. The frequency (2) broadband spectrum will be maximum. The conclusion is the second criterion electromagnetic Harbinger earthquakes.

How will set lower, electric and magnetic fields on the surface of the Earth have characteristic frequency patterns. These patterns will be the third criterion of electromagnetic Harbinger earthquakes. Simultaneous display of all three criteria set out above would constitute a clear manifestation of the electromagnetic Harbinger earthquakes. Here we note the following. As follows from (2), the range of feasible depths of foci of earthquakes $(h=10 \div 30 \mathrm{~km})$ and values of resistivity of solid rock $\left(\rho=10^{4} \div 10^{5} \mathrm{Ohm} \cdot \mathrm{m}\right)$, the optimal frequency of measurements, should be the order of the $1 \mathrm{~Hz} \div$ $1 \mathrm{kHz}$. In [11] was offered search program of electromagnetic Harbinger at frequencies less than $1 \mathrm{~Hz}$. For example, at a frequency of $0.1 \mathrm{~Hz}$, as follows from (2), the depth of the epicenter of the earthquake should be $500 \mathrm{~km}$. But pockets of the earthquake at such a depth is very rare, compared to centers at a depth $h=10 \div 30 \mathrm{~km}$. Indeed, [12] noted that after years of research, search for electromagnetic Harbinger to earthquakes in the frequency range of less than $1 \mathrm{~Hz}$, have not yielded significant results.

We do not consider here May 24, 2013 in the Centre of the earthquake in the sea of Okhotsk at a depth $600 \mathrm{~km}$. This issue requires a separate scrutiny.

\section{The Formation of Electromagnetic Harbinger}

Recently appeared on the Internet indicate that when the cracking of rocks in outer space occurs a significant emission of electromagnetic energy. This is a direct indication that the below studies are not without foundation. Consider the possibility of detecting the Earth's surface electromagnetic precursor earthquakes. While we assume that such Harbinger exists. Hope this point research [2-7], according to which, when crustal deformation caused by faults or prior, generated electromagnetic signals-electric potential anomalies, change of resistance and other electrical manifestations. In the work [3] it was found that the crack in the Earth's crust is a mechanical converter elastic deformations in the electric dipole Dynamics accompanied by crack and relaxation devices generating electromagnetic pulses. Moreover, as noted in [3], oriented normal to the surface of the Earth electric dipole has charge significantly more than the horizontal radiation source. You must install the electromagnetic signals 
dipole oriented normal to the Earth's surface. To describe the electrical properties of solid Wednesday in radio and Geophysics uses two parameters - conductivity $\sigma(=1 / \rho)$ and permittivity $\varepsilon$. Together these electrical properties lead to the next. If the lithosphere in small domestic sites is in a tense position and this condition leads to the formation of electric shock for a long time, these conditions may not be sufficient to generate electromagnetic fields of such a level that they managed to reach the surface media, where they can be measured. Maxwell's equations with them electrical parameters is called material Maxwell equations [9]. Of them, in uniform media semipermeable (i.e. having conductivity $\sigma$ and permittivity $\varepsilon$ ) current $J$ and charge $Q$ associated with the following equation:

$$
\frac{d Q}{d t}+\frac{\sigma Q}{\varepsilon_{0} \varepsilon}=J \text {. }
$$

In here $\varepsilon_{0}$ - dielectric constant. By adopting the $J=J_{0} \exp (-i \omega t)$, get a general solution to the equation $(3)$ :

$$
Q=\left(Q_{0}-\frac{J_{0}}{-i \omega+\sigma / \varepsilon_{0} \varepsilon}\right) \exp \left(-\frac{\sigma}{\varepsilon_{0} \varepsilon} t\right)+\frac{J_{0} \exp (-i \omega t)}{-i \omega+\sigma / \varepsilon_{0} \varepsilon}
$$

For granitic rocks $\rho \sim 10^{5} \mathrm{Ohm} \cdot \mathrm{m}, \varepsilon=10[9]$, and value $\varepsilon_{0} \varepsilon \rho \sim 10^{-6} \mathrm{~s}$. Small size this time means that appear during mechanical stress charges almost instantly fade. Therefore, first, not periodical term in (4) will be set to zero. When this term with periodic factor remains, i.e.,

$$
Q=\frac{J_{0} \exp (-i \omega t)}{-i \omega+\sigma / \varepsilon_{0} \varepsilon}
$$

we can see that if the current in the source changes with time periodically, the charge does not disappear. This fact leads to the fact that the time may be sufficient to media formed electromagnetic field which can be measured on the surface media. In the above, we have determined that it is necessary to carry out measurements at frequencies of $1 \mathrm{~Hz}$ or higher.

\section{Principle Farm for Lateral Wave}

An example of lateral electromagnetic wave is the radiation dipole, located deep in the Earth's lithosphere (the upper layer of the Earth's crust). According to the Geophysics [1], the lithosphere consists of 6 blocks, which in turn are divided into smaller units. Each of these blocks is split further into smaller blocks, etc. and all this floats on the hot mantle. All large and small units are in perpetual motion. They rub against each other, carving the "spark". Rub may only plane that from a distance look like thin strips. Deep in the lithosphere pile cutting "sparks" look like radiation of electric dipole. When there is a slow movement of blocks their radiation, obviously, you might not consider. But during the preparation of tectonic stress movement of blocks will be fast enough. Electromagnetic radiation can be substantial, it can reach the surface and be measured. This opens the search path electromagnetic Harbinger earthquakes!

Let the dipole is located at a depth $h$ above the point $\mathrm{O}$ on the Earth's surface (Figure 2). Measuring complex is located on the distance $x$ the point $\mathrm{O}$.

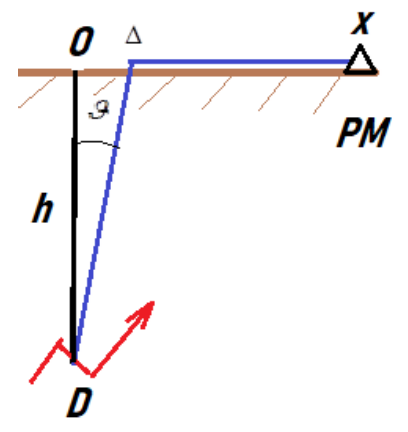

Figure 2. Radiation from the dipole $D$, in the form of lateral wave on toolpath $D \Delta x$ comes at the point of measurement of PM at a distance $x$ from the point $\mathrm{O}$. 
Radiation from the dipole tends to as soon as possible with a speed $v$ to reach the surface at the point $\Delta$ continue to spread through the air at the speed of light to the point of measurement PM. Let the radiation reaches the surface at a distance $\Delta$ from the point of $O$. The total travel time will be

$$
t=\frac{\sqrt{h^{2}+\Delta^{2}}}{v}+\frac{x-\Delta}{c} \text {. }
$$

From the condition that this time the minimum: $t(\Delta)=$ min, i.e. $\frac{d t}{d \Delta}=0$, find

$$
\sin \vartheta=\frac{v}{c}=\frac{\Delta}{\sqrt{h^{2}+\Delta^{2}}} .
$$

The resulting ratio allows you to find a particular point where the calculated integrated Sommerfeld integral known to vector potential. Where simple differentiation can install the components of an electromagnetic field. We have these fields will find another method, first used in [8]. Here we will carry out more detailed course of these calculations.

\section{Scalar and Vector Potentials in Homogeneous Semipermeable Media}

Response media to a semipermeable external electromagnetic field radio describe entering into Maxwell's equations two parameters - conductivity $\sigma$ and permittivity $\varepsilon$. We assume that these same parameters can be described and response media to the electromagnetic fields of nature, arising in the preparation of various stresses in the lithosphere of the Earth. Generally considered that the charge fluctuation in media fades almost instantly. However, the preparation of stress in the lithosphere takes a finite time, and this may be sufficient to generate electromagnetic fields that they managed to reach the Earth's surface.

Radiation of electromagnetic fields can be conveniently described scalar $\varphi$ and vector potential $\vec{A}$. Electric field $\vec{E}$ and magnetic induction $\vec{B}$ expressed through these potentials known formulas $[9,13]$

$$
\vec{B}=\nabla \times \vec{A}, \vec{E}=-\nabla \phi-\frac{\partial \vec{A}}{\partial t}
$$

In here $\nabla$ - del operator, cross $\times$ mean vector multiplication. Vectors $\vec{E}$ and $\vec{B}$ obey the material equations of Maxwell:

$$
\begin{gathered}
\nabla \cdot \varepsilon \vec{E}=\rho / \varepsilon_{0}, \\
c^{2} \nabla \times \vec{B}=\frac{\partial \varepsilon \vec{E}}{\partial t}+\frac{\sigma \vec{E}}{\varepsilon_{0}}+\frac{\vec{j}}{\varepsilon_{0}} .
\end{gathered}
$$

In here $c$ - the speed of light, $\varepsilon_{0}$ - dielectric constant, $\rho$ - charge density, $\vec{j}$ - current density, the point . means a scalar multiplication. The Earth media believe non-magnetic, i.e. believe magnetic permeability equal to unit. Issued only required us to Maxwell's equations, and they are written in SI. Substituting (8) -(10), using the rules of vector analysis, we get:

$$
c^{2} \nabla^{2} A-\varepsilon \frac{\partial^{2} \vec{A}}{\partial t^{2}}=-\frac{\vec{j}}{\varepsilon_{0}}+\frac{\sigma}{\varepsilon_{0}} \frac{\partial \vec{A}}{\partial t}+\nabla\left(c^{2} \nabla \cdot A+\varepsilon \frac{\partial \phi}{\partial t}+\frac{\sigma}{\varepsilon_{0}} \phi\right) .
$$

To this equation contained only vector potential and source fields set condition:

$$
c^{2} \nabla \cdot A+\varepsilon \frac{\partial \phi}{\partial t}+\frac{\sigma}{\varepsilon_{0}} \phi=0 .
$$

As a result, we get the following equation for the vector potential:

$$
\nabla^{2} \vec{A}-\frac{\varepsilon}{c^{2}} \frac{\partial^{2} \vec{A}}{\partial t^{2}}-\mu_{0} \sigma \frac{\partial \vec{A}}{\partial t}=-\mu_{0} \vec{j} .
$$

Here vacuum magnetic constant $\mu_{0}=1 / \varepsilon_{0} c^{2}$.

Substituting (11) into (12) and (13), we get the equation for the scalar potential: 


$$
\nabla^{2} \phi-\frac{\varepsilon}{c^{2}} \frac{\partial^{2} \phi}{\partial t^{2}}-\mu_{0} \sigma \frac{\partial \phi}{\partial t}=-\frac{\rho}{\varepsilon_{0} \varepsilon} .
$$

Pay attention to the identity of the equations (13) and (14). This dignity (12).

\section{The Solution of Equations for Potentials}

There is no need to solve both equations (13) and (14). If you know the solution to the equation (13), out of the equation (12) easy to find

$$
\phi=\frac{1}{\varepsilon} \exp \left(-\frac{\sigma}{\varepsilon_{0} \varepsilon} t\right) \int_{0}^{t} \nabla \cdot \vec{A} \exp \left(\frac{\sigma}{\varepsilon_{0} \varepsilon} t\right) d t .
$$

For monochromatic radiation sources, field and time-dependent potentials as $\exp (-i \omega t)$, where is $\omega$ circular frequency. In this case, equation (13) is assuming the shape of the wave equation with source:

$$
\nabla^{2} \vec{A}+k^{2} \vec{A}=-\mu_{0} \vec{j} \text {. }
$$

In here

$$
k^{2}=\frac{\omega^{2}}{c^{2}}\left(\varepsilon+\frac{i \sigma}{\varepsilon_{0} \omega}\right),
$$

-square wave numbers in media. In free space $\varepsilon=1, \sigma=0$ and $k=\omega / c$.

\section{The Solution of the Wave Equation with Source}

The Poisson equation

$$
\nabla^{2} \phi=-q / \varepsilon_{0}
$$

according to the principle of superposition is the following decision:

$$
\phi=\frac{1}{4 \pi \varepsilon_{0}} \int \frac{q}{r} d V .
$$

In here $q$ will denote the density of charges. Wave equation for a scalar potential, the source is as follows:

$$
\nabla^{2} \phi+k^{2} \phi=-q / \varepsilon_{0} .
$$

It can be solved in different ways. For example, guess. It turns out that the integral expression in (19) need to add exhibitor $\exp (i k r)$. Then the solution of equation (20) will be

$$
\phi=\frac{1}{4 \pi \varepsilon_{0}} \int e^{i k r} \frac{q}{r} d V .
$$

The decision validates a simple swapping it into the original equation (20).

If the charge density $q$ in integral (21) depends on the time as $q \exp (-i \omega t)$, the solution of equation (20) will be:

$$
\phi=\frac{1}{4 \pi \varepsilon_{0}} \int q \frac{\exp (-i \omega t+i k r)}{r} d V .
$$

Now it is clear that the solution to the equation (16) has the following decision, describing the radiation wave:

$$
\vec{A}(\vec{r}, t)=\frac{\mu_{0}}{4 \pi} \int \frac{1}{R} \exp (-i \omega t+i k R) \vec{j}\left(\vec{r}^{\prime}\right) d V^{\prime} .
$$

In here $R=|\vec{r}-\vec{r}|, V$ - the volume occupied by the charges, responsible for radiation. For a linear current $\vec{j}\left(\vec{r}^{\prime}\right) d V^{\prime}=J d l$, where $d l$ - element wire length, $J$-current in the wire, and equation (23) takes the following form:

$$
\vec{A}(\vec{r}, t)=\frac{\mu_{0}}{4 \pi} \int \frac{1}{R} \exp (-i \omega t+i k R) J\left(\vec{r}^{\prime}\right) d l^{\prime} .
$$




\section{Dipole Radiation}

Applies equation (24) to Hertz dipole radiation, which is a conductor of length l, oriented along the $\mathrm{z}$ axis, and $l<<=2 \pi / k$. At such length current $J$ can be considered independent of coordinates. In integral (24) the magnitude of $R$, by virtue $R>>1$, also can be considered constant, and integrating simply to multiply the whole expression in $l$. Eventually non-zero z-component of the vector potential looks like this:

$$
A_{z}(R, t)=\frac{\mu_{0} J l}{4 \pi} \frac{1}{R} \exp (-i \omega t+i k R) .
$$

Convenient multiplier $b=\mu_{0} J l / 4 \pi$ not yet subscribe and exponent $\exp (-i \omega t+i k R)$ designate how $e$. Then

$$
A_{z}=\frac{1}{R} e .
$$

Will consider radiation in the wave zone for which $\mathrm{R}>>\lambda$. In this case $1 / R$ you cannot differentiate. Next, in the spherical coordinate system

$$
\nabla \cdot \vec{A}=\frac{\partial A_{r}}{\partial r}+\frac{1}{r} \frac{\partial A_{\theta}}{\partial \theta},
$$

where the vector $\boldsymbol{A}$ has components $\left(A_{r}, A_{\varphi}, A_{\theta}\right)$. In spherical coordinates $A_{r}=A_{z} \cos \theta, A_{\theta}=A_{z} \sin \theta$, where is $\theta$ - the polar angle, measured from the z axis. Substituting (26) into equation (22), get a scalar potential:

$$
\varphi=\frac{\omega}{k} \frac{1}{R} e \cos \theta
$$

Here omitted components in proportion to $1 / R^{2}$ and do not depend on time $\exp (-i \omega t)$.

Using vector analysis formula in spherical coordinates, of formulas (26) and (28) find non-zero components of electromagnetic fields:

$$
\begin{gathered}
B_{\phi}=\frac{i k}{R} e \sin \theta, \\
E_{r}=\frac{\omega}{k} \frac{1}{R^{2}} e \cos \theta, \\
E_{\theta}=\frac{i \omega}{R} e \sin \theta .
\end{gathered}
$$

Radiation power is as

$$
P=\frac{2 \pi}{\mu_{0}}\left\langle\frac{E_{\theta} B_{\phi}^{*}+E_{\theta}^{*} B_{\phi}}{4}\right\rangle R^{2} \sin \theta d \theta,
$$

where $^{*}$ - complex conjugation, $\langle\ldots\rangle$ - time averaging [14]. Substituting in (32) formula (29) - (31), and integrating $\int_{0}^{\pi} \ldots \frac{d \theta}{2 \pi}$, taking into account all dimensional factors and that the wavenumber $k$ is the complex numbers, we get:

$$
P=\frac{4}{3} \frac{\pi}{\mu_{0}}\left(\frac{\mu_{0} J l}{4 \pi}\right)^{2} \omega \operatorname{Re} k \exp (-2 R \operatorname{Im} k),
$$

where Re and Im - the negation operation real and imaginary parts, respectively. If the radiation occurs in free space, $k=\omega / c$, and from (33) should be known the result [13]:

$$
P=\frac{4}{3} \pi \sqrt{\frac{\varepsilon_{0}}{\mu_{0}}}\left(\frac{\mu_{0} J l}{4 \pi}\right)^{2} \omega^{2} .
$$

See that radiation power in media (33) has a more complicated than frequency dependence of radiated power (34) in free space. 


\section{The Fields on the Surface of the Earth}

Here you need to solve the equation (16) in air media. But you can use the images directly to fields. We will perform a detailed calculation for magnetic induction. Figure 1 presents geometry tasks, $h$ is the depth of the epicenter voltage. From the formula (29) have

$$
\begin{gathered}
B_{\phi}(R, \theta)=\frac{i k}{R} e \sin \theta, \\
B_{\phi}^{\prime}\left(R^{\prime},-\theta\right)=-\frac{i k}{R} e \sin \theta,
\end{gathered}
$$

where is $\vec{R}^{\prime}=\vec{R}-2 \vec{h}$. A common expression on the surface for the tangential components of magnetic induction

$$
B_{\tau}=B_{\varphi}+B_{\varphi}^{\prime}=i k\left(\frac{e}{R}-\frac{e^{\prime}}{|\vec{R}-2 \vec{h}|}\right) \sin \theta .
$$

It should be reminded that $h<<R$, then we get the following:

$$
\begin{gathered}
B_{n}=0, \\
B_{\tau}=\frac{\mu_{0} J l}{4 \pi} \frac{i k h}{R^{2}} \sin 2 \theta \exp (-i \omega t+i k R) .
\end{gathered}
$$

The magnetic field has only horizontal components. Similarly, find the component of the electric field:

$$
\begin{gathered}
E_{n}=\frac{\mu_{0} J}{4 \pi} \frac{4 i \omega h}{k R^{2}} \cos ^{2} \theta \exp (-i \omega t+i k R), \\
E_{\tau}=0 .
\end{gathered}
$$

The electric field has only normal (vertical) component.

It is important to note the following. Actually, in the zone of the hearth earthquakes formed system of cracks of different lengths and oriented randomly. It is therefore necessary to take into account the secondary sources of radiation at the Earth's surface, and to average, randomly oriented dipole emitters. This can be done after detection of the electromagnetic principle Harbinger earthquake.

\section{Conclusion}

The equation for the scalar and vector potentials applied to the task of dipole radiation Hertz, steeped in solid media and oriented normal to the surface of the Earth. It has been established that if the free space is proportional to the square of the dipole radiation power frequencies, the radiation power in Wednesday depends on how frequently more complicated way. Correlations for the components of the electromagnetic field in the wave zone-the first non-null expression series in the inverse degrees of distance from the dipole to the measuring device on the surface of the Earth. These ratios using image method applied to clarify the picture fields on the surface of flat media. It has been established that the approximation on a flat surface magnetic field has only a tangential component, and the electric field is normal component. The have optimum frequency for which you want to measure.

It is shown that if the mechanical stresses in solid media periodically create changing and separated in the space opposite electric charges, these charges do not fade and time is enough to generate electromagnetic field. On the basis of the above, the proposed approach to finding a possible Harbinger of an earthquake. Alternating electric field measured vertical electric antenna. When the measured electrical field strength is proportional to frequency earthquake Harbinger. The tangential magnetic field measured at the Earth's surface, while the measured tension last has a complicated dependence on frequency but does not depend on the orientation of the magnetic antenna. Independence of the measured magnetic field orientation of magnetic antenna allows as a last offer reel the wire on the toroid core to increase the sensitivity of the antenna. Because Harbinger emission spectrum is not known in advance, the measurements must be carried out a broadband receiver. Proposed criteria clearly point to a deep source of radiation of electromagnetic waves from the nascent earthquake. For the implementation of the proposed article approach to finding electromagnetic precursor in earthquake- 
prone regions, you need to create a regional network of monitoring stations. This would allow constant monitoring to predict earthquakes in seismic areas. Having two measuring complex in different points of measurement you will locate the source of the earthquake.

\section{References}

1. Sobolev G. A. and Ponomarev A. V., Physics of Earthquakes and Forerunners (Nauka, Moscow, 2003).

2. Kissin I. G., Fluids in the Earth Crust (Nauka, Moscow, 2009).

3. D'yakonov B. P., Martyshko P. S., Troyanov A. K., et al., Dokl. Akad. Nauk, 430(1), 105 (2010) [Dokl. Earth Sci. 430, 54 (2010)].

4. Bolt B. A., Earthquakes (Freeman, New York, 1999; Mir, Moscow, 1981).

5. Sobolev S. A., Fundamental of Earthquake Prediction (Nauka, Moscow, 1993).

6. Kissin I. G. and Ruzaikin A. I., Fiziks Zemli, No. 7, 67 (2000).

7. Zheligovskii V. A. and Podvigina O. M., Fiziks Zemli, No. 12, 3 (2002).

8. Balkhanov V. K., Bashkuev Yu. B., Way of detecting an electromagnetic earthquake precursor, Technical Physics, 2011, Vol. 56, No 9, pp. 1359-1362. DOI: 10.1134/S1063784211090040.

9. Balkhanov V. K. and. Bashkuev Yu. B, Theoretical Foundations of Surface Resonance Method (BNTs SO RAN, Ulan-Ude, 2005).

10. Bashkuev Yu. B, Electrical Properties of Natural Stratified Media (SO RAN, Novosibirsk, 1996).

11. Hayakawa M., Tomizawa I., Himakura S., Fujinava Y., Takahashi K. and Yishino T. (Phys. Earth Planet. Inter., No. 77, 127, 1993).

12. Verbin Yu. P., Krylov G. N. and Zarkh A. Z. (Usp. Sovr. Radioelektron., No. 1, 62, 2002).

13. Goldshtein L. D. and Zernov N. V., Electromagnetic Fields and Waves (Sovetskoe Radio, Moscow, 1971).

14. Landau L. D. and Lifshitz E. M., Course of Theoretical Physics, Vol. 8: Electrodynamics of Continuous Media (Nauka, Moscow, 1982; Pergamon, New York, 1984). 